\title{
Graphene on transition-metal dichalcogenides: A platform for proximity spin-orbit physics and optospintronics
}

\author{
Martin Gmitra and Jaroslav Fabian \\ Institute for Theoretical Physics, University of Regensburg, 93040 Regensburg, Germany
}

(Received 13 July 2015; published 5 October 2015)

\begin{abstract}
Hybrids of graphene and two-dimensional transition-metal dichalcogenides (TMDCs) have the potential to bring graphene spintronics to the next level. As we show here by performing first-principles calculations of graphene on monolayer $\mathrm{MoS}_{2}$, there are several advantages of such hybrids over pristine graphene. First, Dirac electrons in graphene exhibit a giant global proximity spin-orbit coupling, without compromising the semimetallic character of the whole system at zero field. Remarkably, these spin-orbit effects can be very accurately described by a simple effective Hamiltonian. Second, the Fermi level can be tuned by a transverse electric field to cross the $\mathrm{MoS}_{2}$ conduction band, creating a system of coupled massive and massless electron gases. Both charge and spin transport in such systems should be unique. Finally, we propose to use graphene/TMDC structures as a platform for optospintronics, in particular, for optical spin injection into graphene and for studying spin transfer between TMDCs and graphene.
\end{abstract}

DOI: 10.1103/PhysRevB.92.155403

PACS number(s): $72.80 . \mathrm{Vp}, 72.25 . \mathrm{Fe}$

\section{INTRODUCTION}

Graphene spintronics [1] has relied exclusively on electrical spin injection [2-4]. Combining graphene with semiconducting two-dimensional transition-metal dichalcogenides (TDMCs) [5] can open new venues for spintronics applications [6,7]. Indeed, TMDCs are becoming increasingly popular in optoelectronics as sensitive photodetectors [8] or, forming lateral heterostructures $[9,10]$, as two-dimensional (2D) solar cells [11]. Important, TMDCs have a sizable spin-orbit coupling and lack space inversion symmetry. As a result, their band structure [12] allows for a valley-resolved optical spin excitation by circularly polarized light [13-15]. TMDCs can thus facilitate optical spin injection into graphene, in hybrid structures.

Efficient growth of $\mathrm{MoS}_{2}$ on graphene has already been demonstrated [16-18]. It was reported that graphene on $\mathrm{MoS}_{2}$ is ultraflat, having large mean free paths [19]; angle-resolved photoemission found an intact Dirac point but a strong hybridization elsewhere in the $\pi$ system [20]. Technological potentials for these hybrid structures are already being discussed [21], mainly as a basis for nonvolatile memory [22], sensitive photodetection [23], and gate-tunable persistent photoconductivity [24]. Recently, the spin Hall effect in graphene on few-layer $\mathrm{WS}_{2}$ was observed at room temperature [25].

In this paper we establish, by first-principles calculations, fundamental electronic properties and the spin-orbit fine structure of the graphene Dirac bands for graphene on monolayer $\mathrm{MoS}_{2}$, and introduce an effective spin-orbit Hamiltonian which explains the proximity induced spin splittings of the Dirac states. We show that the induced spin-orbit coupling is giant, being 20 times more than in pristine graphene. We also discuss the field effect on the band offsets of the two materials. Finally, we present possible experimental schemes to perform optical spin injection into graphene and study spin tunneling from TMDCs through graphene.

\section{FIRST-PRINCIPLES RESULTS}

To establish the electronic and spin properties of graphene on $\mathrm{MoS}_{2}$ we used first-principles methods based on density- functional theory [26]. Structural relaxation and electronic structure calculations were performed with QUANTUM ESPRESSO [27], using norm conserving pseudopotentials with a kinetic energy cutoff of $60 \mathrm{Ry}$ for wave functions. For the exchange-correlation potential we used the generalized gradient approximation [28]. To reduce structural strain we constructed a large supercell of 59 atoms, comprising a $3 \times 3$ supercell of $\mathrm{MoS}_{2}$ and a $4 \times 4$ supercell of graphene, with a residual lattice mismatch of $1.4 \%$. Atomic positions were relaxed using the quasi-Newton algorithm based on the trust radius procedure, including the van der Waals interaction, which was treated within a semiempirical approach [29,30]. The relaxed interlayer distance between graphene and $\mathrm{MoS}_{2}$ is $3.37 \AA$ [see Fig. 1(a)]. Such a quasicommensurate superstructure of TMDC has been grown on highly oriented pyrolytic graphite (HOPG) [31]. In this supercell the $K$ point of $\mathrm{MoS}_{2}$ is mapped to the $\Gamma$ point in the reduced Brillouin zone. The calculated electronic band structure is shown in Fig. 1(b). The Dirac cones of graphene are nicely preserved, with the projected Dirac point (which is also the Fermi level) being slightly below the conduction band edge of $\mathrm{MoS}_{2}$. The closeness of the Dirac point to the conduction band of $\mathrm{MoS}_{2}$ enhances screening, which can substantially increase the mean free path in the graphene layer, as recently shown experimentally [19]. We note that use of the dipole correction [32] turned out to be crucial to get proper band offsets between the Dirac point and the conduction band minimum of $\mathrm{MoS}_{2}$. The reduced Brillouin zone was sampled with $12 \times 12 k$ points.

The band offsets between graphene and $\mathrm{MoS}_{2}$ can be controlled by an external electric field applied transverse to the layers [33]. This is demonstrated by our first-principles calculations in Fig. 2(a), where we present $\Delta_{c}$, the difference between the conduction band minima of $\mathrm{MoS}_{2}$ and graphene. At negative fields (pointing towards $\mathrm{MoS}_{2}$ ) the offset increases, leaving both layers neutral. However, positive fields shift the Dirac point above the conduction band minimum of $\mathrm{MoS}_{2}$ and populate graphene with holes and $\mathrm{MoS}_{2}$ with electrons. The Fermi level crosses both the valence band of graphene and the conduction band of $\mathrm{MoS}_{2}$ [see Figs. 2(b) and 2(c)]. This field effect can establish a unique system in which massless 


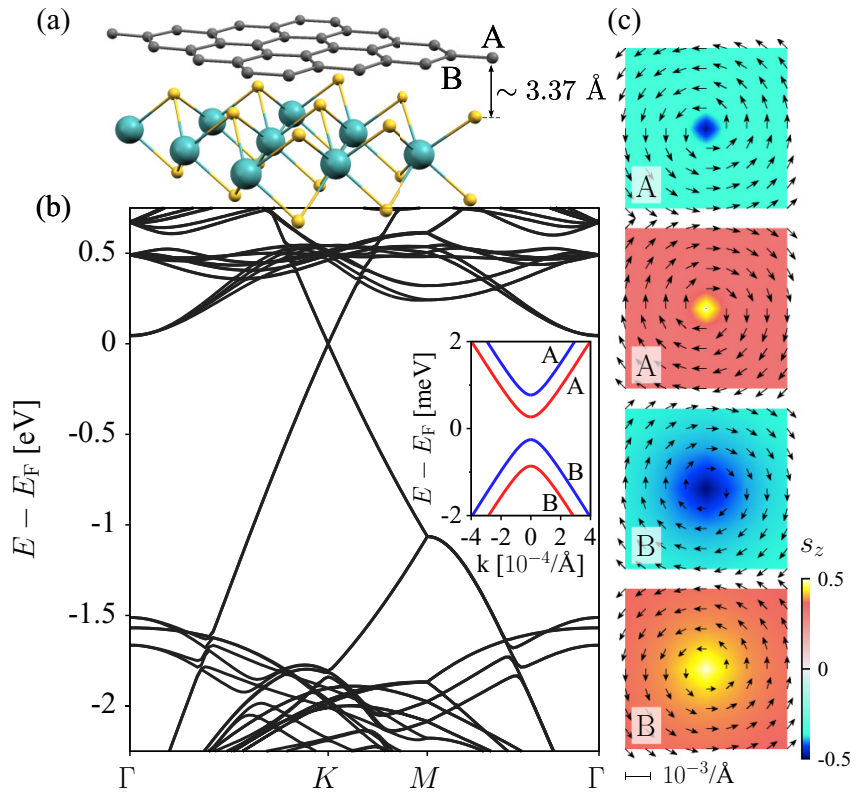

FIG. 1. (Color online) Calculated electronic and spin properties of graphene on two-dimensional $\mathrm{MoS}_{2}$. (a) The supercell used in first-principles calculations. (b) Calculated band structure along high symmetry lines. The inset is a zoom to the fine structure of the low energy bands at the Fermi level, around the Dirac point. Bands with a positive (negative) $z$ component of the spin are shown in red (blue). The sublattice character $(A$ and $B)$ is also indicated. (c) Spin textures for the four bands of the inset in (b).

Dirac electrons are coupled with a conventional 2D electron gas [34].

We now zoom in on the Dirac point at $K$ to see how the electronic spectrum of graphene deforms in the presence of $\mathrm{MoS}_{2}$. This fine structure is shown in the inset to Fig. 1(b). There are two important effects: First, an orbital band gap opens, due to the breaking of the graphene pseudospin symmetry. On average, atoms $A$ and $B$ in the graphene supercell see a different environment coming from the $\mathrm{MoS}_{2}$ layer. This orbital gap is there even in the absence of spin-orbit coupling. It arises from the effective staggered potential induced by the pseudospin symmetry breaking. Second, spin-orbit coupling combined with the broken space inversion symmetry lifts the spin degeneracy of the Dirac valence and conduction bands and leads to the appearance of four distinct bands. This splitting is on the meV scale, which is giant when compared to the $24 \mu \mathrm{eV}$ spin-orbit splitting in pristine graphene [35]. The inset also shows the orbital character of the bands at $K$ : While the valence states are formed at the $B$ sublattice, the conduction states live on $A$. The same orbital ordering is at $K^{\prime}$.

Another important characteristic of the Dirac states is their spin texture. This is plotted in Fig. 1(c) for the four bands from the inset of Fig. 1(b). Directly at $K$ the spins are pointing out of the graphene plane, alternating up and down. Increasing the momentum away from $K$, the spins acquire a winding in-plane component, either clockwise or counterclockwise, suggestive of the strong Rashba effect. At $K^{\prime}$ the spins are reversed.
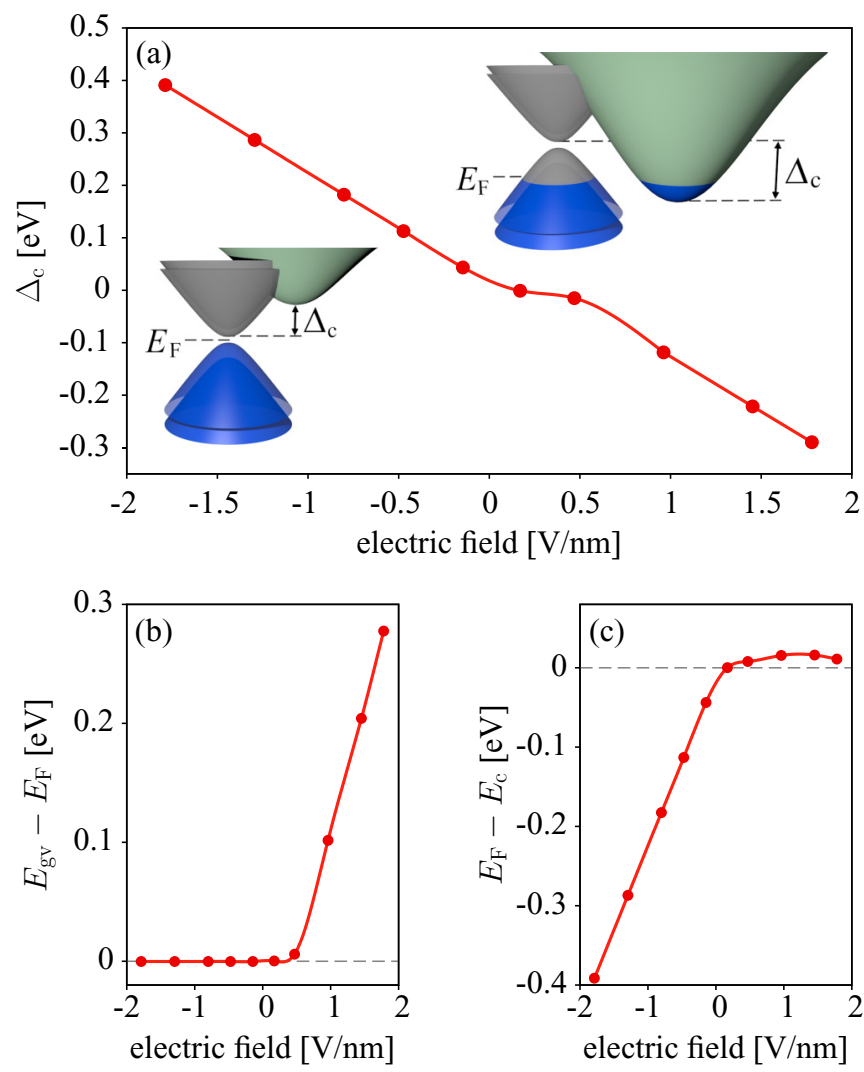

FIG. 2. (Color online) Field effect on graphene/MoS 2 . (a) Calculated offset $\Delta_{c}$ from the conduction band minimum of $\mathrm{MoS}_{2}$ to graphene, as a function of an applied transverse electric field. At positive fields electrons are transferred from graphene to $\mathrm{MoS}_{2}$, establishing a massless-massive electrons bilayer. (b) Net Fermi energy for graphene, as the difference of the valence band maximum $E_{\mathrm{gv}}$ of graphene and the system Fermi level $E_{\mathrm{F}}$. At positive fields the valence band of graphene becomes populated. (c) Net Fermi energy for $\mathrm{MoS}_{2}$, as the difference between the system Fermi level $E_{\mathrm{F}}$ and the conduction band minimum $E_{\mathrm{c}}$ of $\mathrm{MoS}_{2}$. The conduction band of $\mathrm{MoS}_{2}$ becomes populated at positive field, reflecting the population of holes in graphene in (b) as the whole system is neutral.

\section{EFFECTIVE HAMILTONIAN}

Can we understand these proximity-induced changes in graphene's band structure from an effective model? The answer is not obvious since not only do sublattices $A$ and $B$ differ, but even the sites that belong to the same sublattice see different local environments in the supercell. Surprisingly, an effective symmetry-based Hamiltonian with graphene orbitals in the presence of pseudospin inversion symmetry breaking gives a remarkably good description. The model builds on the orbital Hamiltonian for pristine graphene which, close to $K$ $\left(K^{\prime}\right)$ points, is

$$
\mathcal{H}_{0}=\hbar v_{\mathrm{F}}\left(\kappa \sigma_{x} k_{x}+\sigma_{y} k_{y}\right)
$$

Here, $v_{\mathrm{F}}$ is the Fermi velocity of graphene, $k_{x}$ and $k_{y}$ are the Cartesian components of the electron wave vector measured from $K\left(K^{\prime}\right)$, parameter $\kappa=1(-1)$ for $K\left(K^{\prime}\right)$, and $\sigma_{x}$ and $\sigma_{y}$ are the pseudospin Pauli matrices acting on the two-dimensional vector space formed by the two triangular 
sublattices of graphene. Hamiltonian $\mathcal{H}_{0}$ describes gapless Dirac states with the conical dispersion $\varepsilon_{0}=v \hbar v_{\mathrm{F}}|\boldsymbol{k}|$ near the Dirac points; $v=1(-1)$ for the conduction (valence) band.

The staggered potential describing the effective orbital energy difference on $A$ and $B$ sublattices of graphene on $\mathrm{MoS}_{2}$ enters via the Hamiltonian,

$$
\mathcal{H}_{\Delta}=\Delta \sigma_{z} s_{0},
$$

where $\sigma_{z}$ is the pseudospin Pauli matrix and $s_{0}$ is the unit spin matrix; $\Delta$ is the proximity induced gap of the Dirac spectrum. Another consequence of the pseudospin inversion asymmetry is the sublattice-resolved intrinsic spin-orbit coupling. Indeed, the intrinsic coupling acts solely on a given sublattice: It is a next-nearest-neighbor hopping [36]. We describe it in our model with parameters $\lambda_{\mathrm{I}}^{\mathrm{A}}$ and $\lambda_{\mathrm{I}}^{\mathrm{B}}$ for sublattices $A$ and $B$, respectively. The corresponding proximity induced spin-orbit coupling Hamiltonian close to $K\left(K^{\prime}\right)$,

$$
\mathcal{H}_{\text {SO }}=\lambda_{\mathrm{I}}^{\mathrm{A}}\left[\left(\sigma_{z}+\sigma_{0}\right) / 2\right] \kappa s_{z}+\lambda_{\mathrm{I}}^{\mathrm{B}}\left[\left(\sigma_{z}-\sigma_{0}\right) / 2\right] \kappa s_{z},
$$

is a generalization of the McClure-Yafet Hamiltonian for graphene [1,37]. A similar Hamiltonian has been derived for hydrogenated graphene where pseudospin symmetry is explicitly broken [38]. We denote by $s_{z}$ the spin Pauli matrix, while by $\sigma_{0}$ the unit matrix acting on the pseudospin (sublattice) space. If $\lambda_{\mathrm{I}}^{\mathrm{A}}=\lambda_{\mathrm{I}}^{\mathrm{B}}$, the main effect of the intrinsic spin-orbit coupling is to enhance the anticrossing of the pristine graphene Dirac cones [35], leaving the spin degeneracy intact. However, if $\lambda_{\mathrm{I}}^{\mathrm{A}} \neq \lambda_{\mathrm{I}}^{\mathrm{B}}$, as in our case, the spin degeneracy gets lifted by this intrinsic term already, reflecting the loss of space inversion symmetry.

Placing graphene on $\mathrm{MoS}_{2}$ also breaks the lateral mirror symmetry, giving rise to the Rashba type spin-orbit coupling [36],

$$
\mathcal{H}_{\mathrm{R}}=\lambda_{\mathrm{R}}\left(\kappa \sigma_{x} s_{y}-\sigma_{y} s_{x}\right),
$$

where $\lambda_{\mathrm{R}}$ is the Rashba parameter and $s_{x}, s_{y}$ are the spin Pauli matrices. In the hopping language, the Rashba coupling is the nearest-neighbor spin-flip hopping, contributing further to the spin splitting of the bands, and defining the spin quantization axis for each Bloch state, away from the time reversal points $\Gamma$ and $M$.

Hamiltonian $\mathcal{H}_{0}+\mathcal{H}_{\Delta}+\mathcal{H}_{\mathrm{SO}}+\mathcal{H}_{\mathrm{R}}$ fully describes graphene's bands at $K\left(K^{\prime}\right)$. Its eigenenergies are

$$
\begin{aligned}
\varepsilon_{v \mu}= & \frac{1+v \mu}{2}\left[v \Delta+\frac{1+v}{2} \lambda_{\mathrm{I}}^{\mathrm{A}}+\frac{1-v}{2} \lambda_{\mathrm{I}}^{\mathrm{B}}\right] \\
& -\frac{1-v \mu}{4}\left[\lambda_{\mathrm{I}}^{\mathrm{A}}+\lambda_{\mathrm{I}}^{\mathrm{B}}-v \sqrt{\left(2 \Delta-\lambda_{\mathrm{I}}^{\mathrm{A}}+\lambda_{\mathrm{I}}^{\mathrm{B}}\right)^{2}+16 \lambda_{\mathrm{R}}^{2}}\right],
\end{aligned}
$$

where $\mu=1$ ( -1 ) for spin up (down) branches. The expectation values of the spin along $z$ for the corresponding states are given by

$$
\begin{aligned}
& \left\langle s_{z}\right\rangle_{v \mu} \\
& =\frac{\mu \kappa \hbar}{2}\left[\frac{1+\nu \mu}{2}+\frac{1-v \mu}{2} \frac{2 \Delta-\lambda_{\mathrm{I}}^{\mathrm{A}}-\lambda_{\mathrm{I}}^{\mathrm{B}}}{\sqrt{\left(2 \Delta-\lambda_{\mathrm{I}}^{\mathrm{A}}+\lambda_{\mathrm{I}}^{\mathrm{B}}\right)^{2}+16 \lambda_{\mathrm{R}}^{2}}}\right] .
\end{aligned}
$$
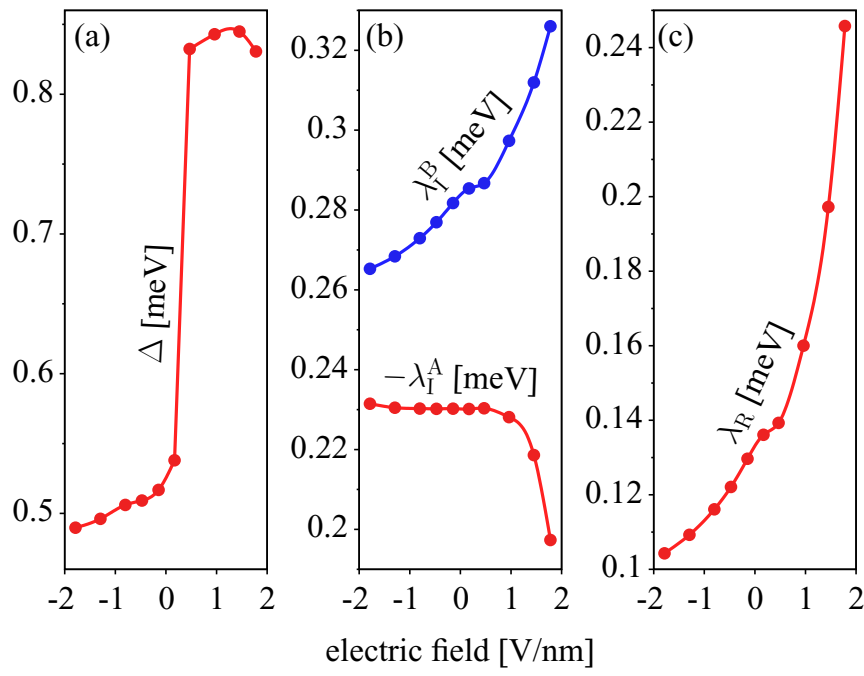

FIG. 3. (Color online) Calculated effective Hamiltonian parameters at the $K$ point: (a) Hybridization gap $\Delta$, (b) sublattice-resolved intrinsic spin-orbit coupling $\lambda_{\mathrm{I}}^{\mathrm{A}}$ and $\lambda_{\mathrm{I}}^{\mathrm{B}}$ (they have opposite signs), and (c) Rashba parameter $\lambda_{R}$, as functions of the applied transverse electric field.

Using the formulas for the eigenenergies, Eq. (5), and for the spin expectation values, Eq. (6), we can algebraically extract the orbital band gap $\Delta$ and the three spin-orbit parameters $\lambda_{\mathrm{I}}^{\mathrm{A}}, \lambda_{\mathrm{I}}^{\mathrm{B}}$, and $\lambda_{\mathrm{R}}$ by comparing to our first-principles data for the fine structure at $K$ [see the inset to Fig. 1(b)]. The extracted parameters are shown in Fig. 3 as a function of the applied transverse electric field. The orbital proximity gap $\Delta$ is about $0.5 \mathrm{meV}$ in zero field. In fields greater than $0.5 \mathrm{~V} / \mathrm{nm}$, the gap exhibits a steep increase, which is related to the transfer of the electronic charge from graphene to $\mathrm{MoS}_{2}$ (see Fig. 2). The proximity spin-orbit parameters in Figs. 3(b) and 3(c) are about $0.2 \mathrm{meV}$, which is 20 times more than in pristine graphene [35]. Similar giant values of spin-orbit coupling in graphene are induced by hydrogen adatoms [38-40], and even more by fluorine [41], though the mechanisms are different. Unlike in the adatom cases in which the induced spin-orbit coupling is only local, in our case the giant coupling is global. While the intrinsic parameters $\lambda_{\mathrm{I}}^{\mathrm{A}}$ and $\lambda_{\mathrm{I}}^{\mathrm{B}}$ change rather moderately with applying the electric field, the Rashba parameter $\lambda_{R}$ [see Fig. 3(c)] more than doubles in increasing the field from -2 to $2 \mathrm{~V} / \mathrm{nm}$.

What is the origin of the induced giant spin-orbit coupling in graphene on $\mathrm{MoS}_{2}$ ? We trace the enhancement to the hybridization of the carbon orbitals with the $d$ orbitals of Mo. We find only $0.3 \%$ of $d$ orbitals at the $K$ point by analyzing the calculated density of states. But when we turn off the spin-orbit coupling on Mo atoms in the supercell, the orbital gap in zero field remains almost unchanged $(\Delta=0.506 \mathrm{meV})$, while the spin-orbit parameters drop to their pristine graphene values $\lambda_{\mathrm{I}}^{\mathrm{A}}=24 \mu \mathrm{eV}, \lambda_{\mathrm{I}}^{\mathrm{B}}=23 \mu \mathrm{eV}$, and $\lambda_{\mathrm{R}}=10 \mu \mathrm{eV}$, which are, curiously, also determined by $d$ orbitals, but from carbon atoms [35].

Away from $K\left(K^{\prime}\right)$, the spin splittings depend on the momentum. In order to describe our first-principles data, we add the pseudospin inversion asymmetry (PIA) spin-orbit 
coupling term [38] which, as the intrinsic coupling, represents the next-nearest-neighbor hopping, but with a spin flip. The full model Hamiltonian describes the data perfectly [42].

Although our effective model should capture the basic physics of graphene on TMDCs, the extracted parameters are for the specific supercell of graphene on $\mathrm{MoS}_{2}$. Certainly, taking an even larger cell that could further reduce strain, or twisting the two layers as could happen in experiments, would lead to a different set of parameters, although the orders of magnitudes would likely stay. In a macroscopic experimental structure we expect moiré patterns which would transform our Hamiltonian into a Hamiltonian density, with an orbital gap and spin-orbit fields, perhaps even averaging some of the parameters (such as $\Delta$ and the difference between $\lambda_{\mathrm{I}}^{\mathrm{A}}$ and $\lambda_{\mathrm{I}}^{\mathrm{B}}$ ) to zero. Our extracted parameters can then be viewed as effective standard deviations of the spatial variations, suitable as input for charge and spin transport model calculations for such samples. We also expect that graphene on TMDCs could produce superlattice features as in graphene on hexagonal boron nitride (hBN) [43].

\section{OPTOSPINTRONICS}

We propose graphene-TMDC hybrids, such as the one studied above based on $\mathrm{MoS}_{2}$, as an ideal platform for optospintronics. In Fig. 4(a) we give an optical spin injection scheme into graphene. A circularly polarized light, tuned to the band gap of TMDC, excites electron spins by optical orientation $[6,44]$. In effect, the light produces spin-polarized excitons which dissociate into spin-polarized electrons and holes. As in the recent optical experiment [24], we expect that electrons will be transferred to graphene, leaving holes behind in TMDC, although in which way electrons and holes split may depend on the TMDC material as well as on gating. The spin-polarized electrons (or holes) diffuse in graphene. One can detect this spin accumulation either optically, by observing a circular polarization of the photoluminescence [44] elsewhere in graphene on TMDC, or electrically, or using Kerr spectroscopy [45]. The latter is illustrated in Fig. 4(a): A ferromagnetic electrode on top of graphene detects the presence of the spin accumulation in graphene [6,7]. Spin precession in graphene can be observed as the Hanle signal (which is not possible to see in the spinvalley coupled TMDC [46]), by applying an external magnetic field transverse to the injected spin, providing Larmor precession [7].

Spin transport per se in graphene-TMDC bilayers should be fascinating. The presence of the giant, effectively uniform spin-orbit fields should give large spin Hall signals, even greater than in hydrogenated graphene [40]. Most important, as our calculations show, the spin, as the charge, properties of these structures are expected to be highly field tunable. The fascinating prospect of realizing the massive-massless electron gas coupling of the two electron gases, if the Fermi level is positioned in both band structures, calls for new theories of spin transport and spin relaxation in such hybrid systems.

To demonstrate spin tunneling from a TMDC through graphene one could use a sandwich structure, as pictured (a)

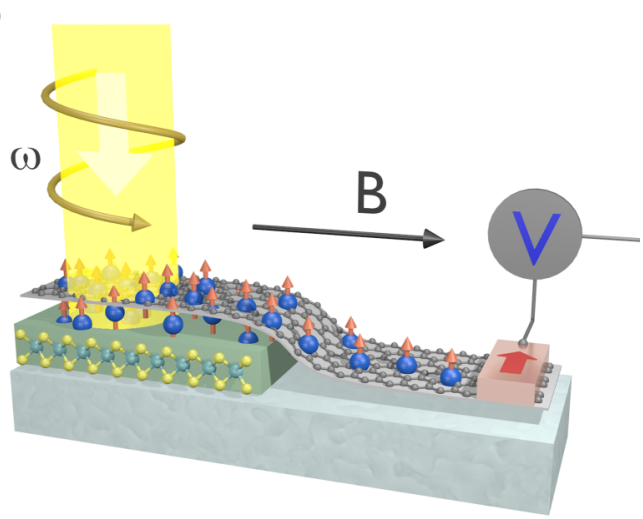

(b)

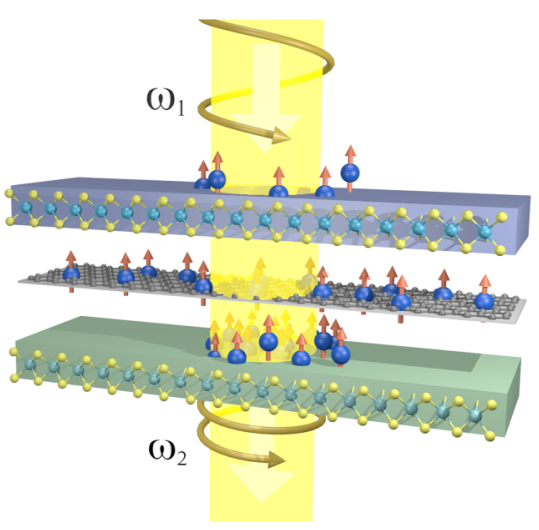

FIG. 4. (Color online) Optospintronic schemes for grapheneTMDC hybrids. (a) Optical spin injection into graphene, facilitated by the semiconducting TMDC. A circularly polarized light excites spin-polarized electrons in the semiconductor. The spin is transferred to graphene where it can be detected as a Hanle signal by the ferromagnetic electrode. (b) Spin transfer between two different TMDCs, encapsulating graphene. Circularly polarized light tuned to the band gap of the top material excites electron spins which can tunnel to the lower material, exhibiting a circular luminescence peaked at its band gap frequency.

in Fig. 4(b). The two semiconductors have different band gaps, allowing one to discern the photoluminescence signals from the top and bottom layers. If the spin pumping light is tuned to the band gap of the top layer, the spin-polarized carriers would be excited there and would tunnel through graphene to the bottom layer, in which they would recombine and emit circularly polarized light with the frequency characteristics of the bottom material. One can envision influencing the signal with a transverse magnetic field, allowing for a Hanle effect. Another possibility is to measure the accumulated spin in the bottom layer using the magnetooptical Kerr effect, as in recent experiments on monolayer $\mathrm{MoS}_{2}$ [47].

\section{CONCLUSION}

We have established, by first-principles calculations, a strong effect of $\mathrm{MoS}_{2}$ on the spin properties of graphene, predicting a giant and field-tunable proximity spin-orbit coupling for Dirac electrons. We have introduced an effective 
spin-orbit Hamiltonian to describe the electronic states around the Fermi level, fitting perfectly the first-principles data. We have also showed that gating can tune the band offsets of the two layers, allowing one to realize a unique system of coupled massless and massive electron gases. Finally, we have proposed to use graphene on TMDCs as a platform for optospintronics with graphene-based two-dimensional material structures.

\section{ACKNOWLEDGMENTS}

We thank T. Korn, C. Schüller, C. Stampfer, B. Beschoten, T. Müller, and B. Özyilmaz for useful discussions and hints regarding possible experimental realizations of optospintronics with graphene-TMDC structures. This work was supported by Deutsche Forschungsgemeinschaft SFB 689, GRK 1570, and by the EU Seventh Framework Programme under Grant Agreement No. 604391 Graphene Flagship.
[1] W. Han, R. K. Kawakami, M. Gmitra, J. Fabian, M. Gmitra, R. K. Kawakami, and W. Han, Nat. Nanotechnol. 9, 794 (2014).

[2] N. Tombros, C. Józsa, M. Popinciuc, H. T. Jonkman, and B. J. van Wees, Nature (London) 448, 571 (2007).

[3] K. Pi, W. Han, K. M. McCreary, A. G. Swartz, Y. Li, and R. K. Kawakami, Phys. Rev. Lett. 104, 187201 (2010).

[4] T.-Y. Yang, J. Balakrishnan, F. Volmer, A. Avsar, M. Jaiswal, J. Samm, S. R. Ali, A. Pachoud, M. Zeng, M. Popinciuc et al., Phys. Rev. Lett. 107, 047206 (2011).

[5] K. F. Mak, C. Lee, J. Hone, J. Shan, and T. F. Heinz, Phys. Rev. Lett. 105, 136805 (2010).

[6] I. Žutić, J. Fabian, and S. Das Sarma, Rev. Mod. Phys. 76, 323 (2004).

[7] J. Fabian, A. Matos-Abiague, C. Ertler, P. Stano, and I. Žutić, Acta Phys. Slovaca 57, 565 (2007).

[8] O. Lopez-Sanchez, D. Lembke, M. Kayci, A. Radenovic, and A. Kis, Nat. Nanotechnol. 8, 497 (2013).

[9] C. Huang, S. Wu, A. M. Sanchez, J. J. P. Peters, R. Beanland, J. S. Ross, P. Rivera, W. Yao, D. H. Cobden, and X. Xu, Nat. Mater. 13, 1096 (2014).

[10] C.-H. Lee, G.-H. Lee, A. M. van der Zande, W. Chen, Y. Li, M. Han, X. Cui, G. Arefe, C. Nuckolls, T. F. Heinz et al., Nat. Nanotechnol. 9, 676 (2014).

[11] A. Pospischil, M. M. Furchi, and T. Mueller, Nat. Nanotechnol. 9, 257 (2014).

[12] A. Kormányos, G. Burkard, M. Gmitra, J. Fabian, V. Zólyomi, N. D. Drummond, and V. Fal'ko, 2D Mater. 2, 022001 (2015).

[13] D. Xiao, G.-B. Liu, W. Feng, X. Xu, and W. Yao, Phys. Rev. Lett. 108, 196802 (2012).

[14] K. F. Mak, K. He, J. Shan, and T. F. Heinz, Nat. Nanotechnol. 7, 494 (2012).

[15] H. Zeng, J. Dai, W. Yao, D. Xiao, and X. Cui, Nat. Nanotechnol. 7, 490 (2012).

[16] Y.-C. Lin, N. Lu, N. Perea-Lopez, J. Li, Z. Lin, X. Peng, C. H. Lee, C. Sun, L. Calderin, P. N. Browning et al., ACS Nano 8, 3715 (2014).

[17] M.-Y. Lin, C.-E. Chang, C.-H. Wang, C.-F. Su, C. Chen, S.-C. Lee, and S.-Y. Lin, Appl. Phys. Lett. 105, 073501 (2014).

[18] A. Azizi, S. Eichfeld, G. Geschwind, K. Zhang, B. Jiang, D. Mukherjee, L. Hossain, A. F. Piasecki, B. Kabius, J. A. Robinson et al., ACS Nano 9, 4882 (2015).

[19] C.-P. Lu, G. Li, K. Watanabe, T. Taniguchi, and E. Y. Andrei, Phys. Rev. Lett. 113, 156804 (2014).
[20] H. Coy Diaz, J. Avila, C. Chen, R. Addou, M. C. Asensio, and M. Batzill, Nano Lett. 15, 1135 (2015).

[21] N. A. Kumar, M. A. Dar, R. Gul, and J. Baek, Mater. Today 18, 286 (2015)

[22] S. Bertolazzi, D. Krasnozhon, and A. Kis, ACS Nano 7, 3246 (2013).

[23] W. Zhang, C.-P. Chuu, J.-K. Huang, C.-H. Chen, M.-L. Tsai, Y.-H. Chang, C.-T. Liang, Y.-Z. Chen, Y.-L. Chueh, J.-H. He et al., Sci. Rep. 4, 3826 (2014).

[24] K. Roy, M. Padmanabhan, S. Goswami, T. P. Sai, G Ramalingam, S. Raghavan, and A. Ghosh, Nat. Nanotechnol. 8, 826 (2013).

[25] A. Avsar, J. Y. Tan, T. Taychatanapat, J. Balakrishnan, G. K W. Koon, Y. Yeo, J. Lahiri, A. Carvalho, A. S. Rodin, E. C. T. O'Farrell et al., Nat. Commun. 5, 4875 (2014).

[26] J. Hohenberg and W. Kohn, Phys. Rev. 136, B864 (1964).

[27] P. Giannozzi et al., J. Phys.: Condens. Matter 21, 395502 (2009).

[28] J. P. Perdew, K. Burke, and M. Ernzerhof, Phys. Rev. Lett. 77, 3865 (1996).

[29] S. Grimme, J. Comput. Chem. 27, 1787 (2006).

[30] V. Barone, M. Casarin, D. Forrer, M. Pavone, M. Sambi, and A. Vittadini, J. Comput. Chem. 30, 934 (2009).

[31] M. M. Ugeda, A. J. Bradley, S.-F. Shi, F. H. da Jornada, Y. Zhang, D. Y. Qiu, W. Ruan, S.-K. Mo, Z. Hussain, Z.-X. Shen et al., Nat. Mater. 13, 1091 (2014).

[32] L. Bengtsson, Phys. Rev. B 59, 12301 (1999).

[33] S. Larentis, J. R. Tolsma, B. Fallahazad, D. C. Dillen, K. Kim, A. H. MacDonald, and E. Tutuc, Nano Lett. 14, 2039 (2014).

[34] B. Scharf and A. Matos-Abiague, Phys. Rev. B 86, 115425 (2012).

[35] M. Gmitra, S. Konschuh, C. Ertler, C. Ambrosch-Draxl, and J. Fabian, Phys. Rev. B 80, 235431 (2009).

[36] S. Konschuh, M. Gmitra, and J. Fabian, Phys. Rev. B 82, 245412 (2010).

[37] J. W. McClure and Y. Yafet, in Proceedings of the 5th Conference on Carbon (Pergamon, New York, 1962), Vol. 1, pp. 22-28.

[38] M. Gmitra, D. Kochan, and J. Fabian, Phys. Rev. Lett. 110, 246602 (2013).

[39] A. H. Castro Neto and F. Guinea, Phys. Rev. Lett. 103, 026804 (2009).

[40] J. Balakrishnan, G. Kok, W. Koon, M. Jaiswal, and A. H. Castro Neto, Nat. Phys. 9, 284 (2013). 
[41] S. Irmer, T. Frank, S. Putz, M. Gmitra, D. Kochan, and J. Fabian, Phys. Rev. B 91, 115141 (2015).

[42] See Supplemental Material at http://link.aps.org/supplemental/ 10.1103/PhysRevB.92.155403 for details of calculations of spin expectation values and contributions of pseudospin inversion asymmetry terms to spin-orbit coupling.

[43] R. V. Gorbachev, J. C. W. Song, G. L. Yu, A. V. Kretinin, F. Withers, Y. Cao, A. Mishchenko, I. V. Grigorieva, K. S. Novoselov, L. S. Levitov et al., Science 346, 448 (2014).
[44] F. Meier and B. P. Zakharchenya, Optical Orientation (NorthHolland, New York, 1984).

[45] L. Yang, N. A. Sinitsyn, W. Chen, J. Yuan, J. Zhang, J. Lou, and S. A. Crooker, Nat. Phys., doi: 10.1038/nphys3419.

[46] G. Sallen, L. Bouet, X. Marie, G. Wang, C. R. Zhu, W. P. Han, Y. Lu, P. H. Tan, T. Amand, B. L. Liu et al., Phys. Rev. B 86, 081301 (2012).

[47] G. Plechinger, P. Nagler, Schüller, and T. Korn, arXiv:1404.7674. 